\title{
Analysis of Antennas around NURBS Surfaces by Using a Hybrid Method
}

\author{
Tayfun $O K A N^{1,2}$, Nursel AKÇAM ${ }^{1}$ \\ ${ }^{1}$ Dept. of Electrical-Electronic Engineering, Gazi University, Yukselis Street 5, 06570 Ankara, Turkey \\ ${ }^{2}$ Dept. of Electrical-Electronic Engineering, University of Turkish Aeronautical Association, Okul Street 11, \\ 06790 Ankara, Turkey \\ tayfunokan@gmail.com
}

Submitted January 05, 2018 / Accepted June 22, 2018

\begin{abstract}
To fast and accurately calculate the EM scattering problems like radiation of antenna around complex objects, many different techniques have been performed. However, these techniques (exact solution methods) either have a long calculation time or they (hybrid methods) tend to fail when the antenna is near or adjacent to structures. In this study, to make the scattering analysis of antennas fast and accurately, proposed stationary phase method (SPM) based hybrid MoM-PO method is implemented. For the geometrical modelling part, the antenna and its close vicinity is modelled with triangular facets, and the rest of the structure is modelled with non-uniform rational $b$ spline (NURBS) surfaces which provides efficient and accurate modeling. Maximum $1.7 \mathrm{~dB}$ of difference in the radiation pattern is observed for an antenna positioned around a curvy plate, when the results are obtained by using MoM and the proposed method. Despite that little difference, the calculation time for MoM is around 41 minutes; whereas for the proposed hybrid method it is 9 seconds. Overall, this hybrid formulation and NURBS modeling allow a substantial reduction of computation time and memory requirement to analyze radiation of antennas that are even close or connected to structure surfaces.
\end{abstract}

\section{Keywords}

B-spline surfaces, method of moments (MoM), physical optics (PO), scattering problems, stationary phase method (SPM)

\section{Introduction}

The analyses of radiation in EM problems are mainly focused on 1) the radar cross section (RCS) calculations and 2) the analysis of antennas. Method of moments (MoM) [1] is one of the popular techniques to solve electric field integral equations (EFIE) with high accuracy and reliability, since it is a numerical simulation method. Previously, MoM was used with Rao-Wilton-Glisson (RWG) triangular patch basis function for arbitrary shape objects [2]. For MoM the matrix fill time is $O\left(N^{2}\right)$, where $N$ de- notes the unknowns. So the challenge in this method is the high computational load for high frequencies. As the electrical size of the object increases fast solvers like multilevel fast multipole algorithm (MLFMA) [3] or some asymptotic integration methods like physical optics (PO) are needed.

PO is a high frequency asymptotic method and mostly preferred to calculate the scattered fields of electrically large objects [4], [5]. Although the use of PO causes a loss of accuracy, it is a very efficient technique. Many different methods were developed to calculate the PO current integral, which is a highly oscillatory integral. Most basic ones are developed by Gordon [6] and Ludwig [7]. Some other different calculation approaches have been developed to calculate the current integral, which include Filon method [8], Levin method [9] and numerical steepest descent method [10], [11]. Stationary phase method (SPM) [12-14] is a mathematical approach that takes advantage of the rapidly varying phase and works very fast and accurately for very high frequencies. For SPM, the CPU time is independent of frequency, on the other hand it is not valid in the computation of near field and this generates a problem as an incorrect impedance matrix [13]. In [15] RCS calculation is performed using SPM for trimmed NURBS surfaces.

To overcome the computational complexity of MoM for electrically large structures, hybrid methods combining the MoM with high frequency asymptotic methods are common. In this paper hybrid MoM-PO method is examined for the radiation analysis of antennas that are very close or even connected to electrically large conducting platforms. As both MoM and PO are current-based methods, combining these two seems to be a reasonable choice. This combination simplifies continuity of current on the surface of the structure, which is subdivided into MoM and PO regions. The antenna and its vicinity is analyzed by MoM, the remaining part of the platform is handled by PO. This hybrid method combines unknown MoM currents for some regions, with other surface currents obtained from PO for the rest of the regions. Some contributions applied to $3 \mathrm{D}$ radiation problems can be found in [16], [17] in the past years, where MoM and PO method were combined. But in 
these studies, either the structure was not modeled by NURBS surfaces or the antenna was not close to the structure.

An electromagnetic scattering problem generally involves two issues: geometrical modeling of the object and the scattering analysis of it. NURBS is an efficient technique for modeling, so recently it has been used in many EM problems [12-14], [18], [19]. Small number of patches is used to model complex bodies, so it requires very little memory and computing time. Apart from the above feature, NURBS has many advantages for EM or RCS computations:

1) Object is modeled more precisely.

2) Curvature patches can be used instead of plane facets.

3) Number of patches is lower.

4) Artificial boundaries and edges are not introduced.

5) Requires little computing time and memory.

Although NURBS possesses several advantages, it is not preferred for numerical calculation because of its recursion form. Therefore NURBS surfaces must be transformed into Bezier surfaces through the Cox-De Boor algorithm. In literature MoM calculations are mostly made by using triangular patches, but NURBS based MoM is also applicable [18]. For the NURBS based PO method very fast and efficient results were obtained in the past. Conde et al. employed SPM to calculate PO currents for complex 3-D structures modeled by NURBS surfaces to analyze antenna radiation for bistatic cases [13]. But in this study, it is also recommended to apply hybrid methods to analyze scattering when antenna is very close or connected to the structure. In [20] a fast algorithm is employed to simplify and speed up the locating process of stationary points of PO integrals on b-spline surfaces.

In this study scattering problem of on board antennas is analyzed by using hybrid MoM-PO technique. The structure is subdivided into MoM and PO-regions. MoMregion is modeled by triangular patches and PO-region is modeled using Bézier surfaces. Current work combines MoM and PO technique to analyze antenna around electrically large platform as SPM is used to calculate the PO current integral.

\section{Theoretical Formulations}

\subsection{Definition of the NURBS Surfaces}

The NURBS surface is a parametric surface defined by a set of control points, where a weight value is corresponding to each control point. Mathematical equations for NURBS surfaces are not convenient for numerical calculations. Therefore, defining the NURBS surface by a few rational Bézier surfaces is more common approach to implement [12], [13]. With the help of its Bernstein basic functions, Bézier surfaces are more suitable for numerical calculations. Cox-de Boor algorithm is applied to make the

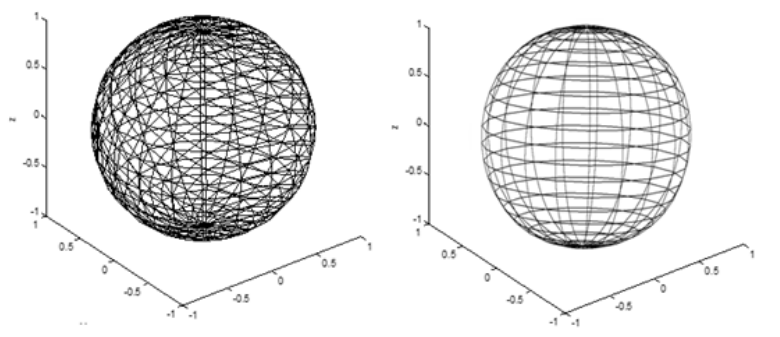

Fig. 1. Sphere modeled by planar triangular patches and modeled with Bézier surfaces.

transformation from NURBS surface to Bézier surfaces [12]. A sphere modeled with triangular patches can be compared with Bézier modeling in Fig. 1.

With Bézier surfaces, the number of patches is lower and for curvature structures a more realistic modeling is possible. The position vector defined by Bézier surface can be given by:

$$
S(u, v)=\frac{\sum_{i=0}^{n} \sum_{j=0}^{p} w_{i, j} p_{i, j} B_{i, n}(u) B_{j, p}(v)}{\sum_{i=0}^{n} \sum_{j=0}^{p} w_{i, j} B_{i, n}(u) B_{j, p}(v)}
$$

where $\boldsymbol{p}_{i, j}$ are the control points, $w_{i, j}$ the weights, $M$ and $N$ are number of control points in $u$ and $v$ directions, with $(u, v) \in[0,1] \times[0,1], B_{i, n}(u)$ and $B_{j, p}(v)$ are the Bernstein polynomials with a formula [12]:

$$
B_{i, n}(t)=\left\{\begin{array}{lr}
\frac{n !}{(n-i) ! i !}(1-t)^{n-i} t^{i}, & 0 \leq i \leq n \\
0, & \text { others }
\end{array}\right.
$$

\subsection{Hybrid MoM-PO Method}

To analyze the radiation of antenna which is positioned close to or even connected to a complex structure, MoM-PO approach is implemented by modifying the impedance matrix of MoM with PO. MoM-PO formulation subdivides the investigated structure into two regions: 1) MoM-region $\left(S_{1}\right)$, where the EFIE is employed and 2) PO-region $\left(S_{2}\right)$, where the magnetic field integral equation (MFIE) is employed. Assuming the current in the MoM-region and in the PO-region is $\boldsymbol{J}^{\mathrm{MoM}}$ and $\boldsymbol{J}^{\mathrm{PO}}$, respectively, as illustrated in Fig. 2.

For hybrid method, the EFIE is set up with $\boldsymbol{J}^{\mathrm{PO}}$ interpreted as source current.

$$
L_{\mathrm{e}}\left(\boldsymbol{J}^{\mathrm{MoM}}\right)+L_{\mathrm{e}}\left(\boldsymbol{J}^{\mathrm{PO}}\right)=-\boldsymbol{E}_{\mathrm{tan}}^{\mathrm{inc}}
$$

where $\boldsymbol{E}_{\text {tan }}^{\text {inc }}$ is the tangential component of the incident electric field.

Similarly, with $\boldsymbol{J}^{\mathrm{MoM}}$ treated as a source current, the MFIE is employed in region $S_{2}$

$$
\boldsymbol{J}^{\mathrm{PO}}=L_{\mathrm{h}}\left(\boldsymbol{J}^{\mathrm{MoM}}\right)+L_{\mathrm{h}}\left(\boldsymbol{J}^{\mathrm{PO}}\right)
$$

where $L_{\mathrm{e}}$ and $L_{\mathrm{h}}$ are the corresponding operators which transform the currents into electric scatter fields and are defined as 


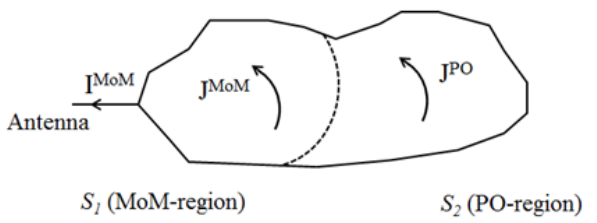

Fig. 2. Antenna connected to a perfect conductor surface, subdivided into $S_{1}$ and $S_{2}$ regions.

$$
\begin{gathered}
L_{\mathrm{e}}(\boldsymbol{J})=-\mathrm{j} \omega \boldsymbol{A}-\nabla \Psi, \\
L_{h}(\boldsymbol{J})=T \hat{\boldsymbol{n}} \times \int_{S} \boldsymbol{J}\left(r^{\prime}\right) \times\left[\nabla^{\prime} G\left(r, r^{\prime}\right)\right] \mathrm{d} S^{\prime}
\end{gathered}
$$

with $\hat{\boldsymbol{n}}$ being the unit outward normal and the parameter $T=2$ for a smooth surface. Vector potential $\boldsymbol{A}$ and scalar potential $\Psi$ are given by

$$
\begin{gathered}
\boldsymbol{A}(r)=\int_{S} \boldsymbol{J}\left(r^{\prime}\right) G\left(r, r^{\prime}\right) \mathrm{d} S^{\prime}, \\
\Psi(r)=-\frac{1}{\mathrm{j} \omega \varepsilon} \int_{S} \nabla^{\prime} \cdot \boldsymbol{J}\left(r^{\prime}\right) G\left(r, r^{\prime}\right) \mathrm{d} S^{\prime}
\end{gathered}
$$

where $\omega$ is the angular frequency, $\boldsymbol{J}\left(r^{\prime}\right)$ is the surface current and $G\left(r, r^{\prime}\right)$ is the free space Green's function

$$
G\left(r, r^{\prime}\right)=\frac{\exp (-\mathrm{j} k R)}{4 \pi R}
$$

$k=2 \pi / \lambda$ is the wavenumber as $\lambda$ is wavelength. $R=\left|\boldsymbol{r}-\boldsymbol{r}^{\prime}\right|$ is the distance between an observation point and source point.

For hybrid MoM-PO approximation, by substituting and using inner product process, (3) and (4) can be transformed into the following matrix equations respectively.

$$
\begin{gathered}
{\left[\boldsymbol{I}_{\mathrm{MoM}}\right]\left[\boldsymbol{Z}_{\mathrm{MoM}}+\boldsymbol{Z}_{\mathrm{MoM}-\mathrm{PO}}^{\mathrm{e}} \boldsymbol{Z}_{\mathrm{MoM}-\mathrm{PO}}^{\mathrm{h}}\right]=\left[\boldsymbol{V}_{\mathrm{MoM}}\right],} \\
{\left[\boldsymbol{I}_{\mathrm{PO}}\right]=\left[\boldsymbol{I}_{\mathrm{PO}}^{\prime}\right]+\left[\boldsymbol{Z}_{\mathrm{MoM}-\mathrm{PO}}^{\mathrm{h}}\right]\left[\boldsymbol{I}_{\mathrm{MoM}}\right]}
\end{gathered}
$$

where $\boldsymbol{I}_{\mathrm{PO}}$ is the current in PO-region, $\boldsymbol{I}_{\mathrm{PO}}$ is the current in the PO region excited by the known external magnetic fields, $\boldsymbol{I}_{\mathrm{MoM}}$ the current in MoM-region, $\boldsymbol{Z}_{\mathrm{MoM}}$ is the impedance matrix in MoM-region, $\boldsymbol{V}_{\mathrm{MoM}}$ is the vector for source voltage in MoM-region, $\boldsymbol{Z}_{\text {MoM-PO }}^{\mathrm{e}}$ and $\boldsymbol{Z}_{\text {MoM-PO }}^{\mathrm{h}}$ are the mutual impedance matrices or the coupling matrices between MoM and PO-regions through the electric field and magnetic field components respectively.

$$
\begin{gathered}
V_{m}=\left\langle W_{m},-\boldsymbol{E}_{\mathrm{tan}}^{\mathrm{inc}}\right\rangle, \\
Z_{m n}^{\eta}=\left\langle W_{m}, L_{\eta}\left(f_{n}\right)\right\rangle, \quad \eta=\mathrm{e}, \mathrm{h} .
\end{gathered}
$$

MoM-PO approach offers tremendous savings in computational resources when the number of unknowns in the MoM-region is significantly smaller than the number of unknowns in the PO-region, or one can say, when the size of the PO-region is much larger than MoM-region. It is also important to note that to yield a valid solution, the current must be continuous across a connected boundary between the regions.

\subsection{PO Approximation Using Stationary Phase Method}

PO approximation gives excellent results when the surface is illuminated, locally flat and edge effects are negligible. To consider the effects of multiple reflections and diffractions, methods like physical theory of diffraction (PTD) or geometrical theory of diffraction (GTD) should be considered. The PO current is defined as

$$
\boldsymbol{J}^{\mathrm{PO}}=\left\{\begin{array}{cc}
2 \hat{\boldsymbol{n}} \times \boldsymbol{H}^{i} & \text { lit } \\
0 & \text { shadow }
\end{array}\right.
$$

where $\hat{\boldsymbol{n}}$ is the outward normal vector of the surface and $\boldsymbol{H}^{\mathrm{i}}$ is the incident magnetic field.

In this work a plane incident wave is under consideration and to fill the impedance matrix the scattered PO field must be solved, which is denoted as $\boldsymbol{E}_{\mathrm{S}}^{\mathrm{PO}}(\boldsymbol{r})$.

$$
\boldsymbol{E}_{\mathrm{S}}^{\mathrm{PO}}(\boldsymbol{r})=\frac{-\mathrm{j}}{\lambda r} \mathrm{e}^{-\mathrm{j} k r} \boldsymbol{E}_{0} \int_{S} \mathrm{e}^{2 \mathrm{j} k \cdot r} \hat{\boldsymbol{k}} \cdot \hat{\boldsymbol{n}} \mathrm{d} s
$$

where $\boldsymbol{E}_{0}$ is the incident electric field at the sampling point, $S$ is the body surface, $\boldsymbol{k}$ is wave vector, $\hat{\boldsymbol{k}}$ is the normalized wave vector.

For a NURBS or Bezier surface, the normal vector and the surface element can be expressed as

$$
\begin{gathered}
\hat{\boldsymbol{n}}=\frac{\boldsymbol{r}_{u} \times \boldsymbol{r}_{v}}{\left|\boldsymbol{r}_{u} \times \boldsymbol{r}_{v}\right|}, \\
\mathrm{d} s=\left|\boldsymbol{r}_{u} \times \overrightarrow{\boldsymbol{r}}_{v}\right| \mathrm{d} u \mathrm{~d} v
\end{gathered}
$$

where $\boldsymbol{r}_{u}=\partial \boldsymbol{r}(u, v) / \partial u$ and $\boldsymbol{r}_{v}=\partial \boldsymbol{r}(u, v) / \partial v$.

Substituting (16) and (17) into (15) gives the following scattered field approximation

$$
\boldsymbol{E}_{\mathrm{S}}^{\mathrm{PO}}(\boldsymbol{r})=\frac{-\mathrm{j}}{\lambda r} \mathrm{e}^{-\mathrm{j} k r} \boldsymbol{E}_{0} \int_{0}^{1} \int_{0}^{1} \mathrm{e}^{2 \mathrm{j} \overrightarrow{\boldsymbol{k}} \cdot \vec{r}(u, v)} \hat{\boldsymbol{k}} \cdot\left(\overrightarrow{\boldsymbol{r}}_{u} \times \overrightarrow{\boldsymbol{r}}_{v}\right) \mathrm{d} u \mathrm{~d} v .
$$

The scattered field is reduced to a calculation of a highly oscillatory integral of the form:

$$
I=\int_{0}^{1} \int_{0}^{1} g(u, v) \mathrm{e}^{\mathrm{j} k f(u, v)} \mathrm{d} u \mathrm{~d} v
$$

where $g(u, v)$ and $f(u, v)$ are called the amplitude function and phase function, respectively.

SPM is a mathematical procedure that solves integrals by just taking the contributions of some critical points distributed over the surface of the structure. The phase term has a stationary behavior and is expanded in Taylor series around the stationary points. As can be seen from Fig. 3, if the surface is plane, $\boldsymbol{n}(r)$ is constant and (19) can be calculated using Gordon's method, if not then SPM is used [13]. There are three different kinds of critical points for this algorithm. 


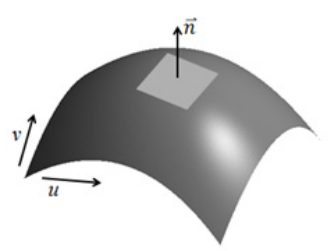

Fig. 3. Stationary phase point on a Bézier surface.

1. The first kind of critical points, these points are found within the surface and they present a stationary behavior in the phase for both $u$ and $v$. Below conditions should be met

$$
\Rightarrow\left\{\begin{array}{l}
f_{u}\left(u_{0}, v_{0}\right)=\left.\frac{\partial f(u, v)}{\partial u}\right|_{u=u_{0}, v=v_{0}}=0 \\
f_{v}\left(u_{0}, v_{0}\right)=\left.\frac{\partial f(u, v)}{\partial v}\right|_{u=u_{0}, v=v_{0}}=0
\end{array}\right.
$$

or

$$
\Rightarrow\left\{\begin{array}{l}
\boldsymbol{k} \cdot \boldsymbol{r}_{u}\left(u_{0}, v_{0}\right)=0 \\
\boldsymbol{k} \cdot \boldsymbol{r}_{v}\left(u_{0}, v_{0}\right)=0
\end{array}\right.
$$

The contribution for the first kind of critical points is given as

$$
\boldsymbol{I}=\frac{\boldsymbol{G}\left(u_{0}, v_{0}\right) \pi \sigma \mathrm{e}^{\mathrm{j} k f\left(u_{0}, v_{0}\right)}}{k \sqrt{\left|f_{u u} f_{v v}-f_{u v}^{2}\right|}}
$$

where

$$
\begin{gathered}
\sigma=\left\{\begin{array}{lr}
\mathrm{j} & \sigma_{1}>0, \sigma_{2}>0 \\
-\mathrm{j} & \sigma_{1}>0, \sigma_{2}<0 \\
1 & \sigma_{1} \sigma_{2}<0
\end{array}\right. \\
f_{u u}\left(u_{0}, v_{0}\right)=\left.\frac{\partial^{2} f(u, v)}{\partial u^{2}}\right|_{u=u_{0}, v=v_{0}} \\
f_{v v}\left(u_{0}, v_{0}\right)=\left.\frac{\partial^{2} f(u, v)}{\partial v^{2}}\right|_{u=u_{0}, v=v_{0}} \\
f_{u v}\left(u_{0}, v_{0}\right)=\left.\frac{\partial^{2} f(u, v)}{\partial u \partial v}\right|_{u=u_{0}, v=v_{0}}
\end{gathered}
$$

$\sigma_{1}$ and $\sigma_{2}$ are the eigenvalues of the matrix $A=\left[\begin{array}{ll}f_{u u} & f_{u v} \\ f_{u v} & f_{v v}\end{array}\right]$.

2. The boundary critical points are the points of the curve bounding the domain of integration on which the below conditions are fulfilled.

$$
\begin{aligned}
& f_{v}\left(0, v_{0}\right)=0, f_{v}\left(1, v_{0}\right)=0, \\
& f_{u}\left(u_{0}, 0\right)=0, f_{u}\left(u_{0}, 1\right)=0,
\end{aligned}
$$

or

$$
\begin{aligned}
& \boldsymbol{k} \cdot \boldsymbol{r}_{u}\left(0, v_{0}\right)=0, \boldsymbol{k} \cdot \boldsymbol{r}_{u}\left(1, v_{0}\right)=0, \\
& \boldsymbol{k} \cdot \boldsymbol{r}_{u}\left(u_{0}, 0\right)=0, \boldsymbol{k} \cdot \boldsymbol{r}_{u}\left(u_{0}, 1\right)=0 .
\end{aligned}
$$

As can be seen from the above equations at each boundary, one coordinate is fixed and the phase function must exhibit a stationary behavior in the remaining coordinate. The contribution of this integral is given as

$$
\boldsymbol{I}=\frac{\mathrm{j}(-1)^{\alpha} \boldsymbol{G}\left(u_{0}, v_{0}\right) \mathrm{e}^{\mathrm{j} k f\left(u_{0}, v_{0}\right)}}{k f_{\alpha}\left(u_{0}, v_{0}\right)} \sqrt{\frac{\mathrm{j} \pi}{k f_{\beta \beta}\left(u_{0}, v_{0}\right)}}
$$

where $\alpha$ is $v$ and $\beta$ is $u$ in the first and second cases; and $\alpha$ is $u$ and $\beta$ is $v$ in the third and fourth cases. When boundary points are close to the first kind of critical points, it is necessary to consider the coupling effect between them. Then the contribution equation becomes

$$
\begin{gathered}
\boldsymbol{I}=\frac{s(-1)^{\alpha}}{k} \boldsymbol{G}\left(u_{0}, v_{0}\right) \mathrm{e}^{\mathrm{j} k f\left(u_{0}, v_{0}\right)} \mathrm{e}^{-\mathrm{j} s v_{\alpha}^{2}} \operatorname{sign}\left(k f_{\alpha}\left(u_{0}, v_{0}\right)\right) \\
\cdot F_{s}\left(v_{\alpha}\right) \sqrt{\frac{\mathrm{j} \pi}{f_{\beta \beta}\left(u_{0}, v_{0}\right) \cdot\left|f_{\alpha \alpha}\left(u_{0}, v_{0}\right)\right|}}
\end{gathered}
$$

where

$$
\begin{gathered}
s=\operatorname{sign}\left(k f_{\alpha \alpha}\left(u_{0}, v_{0}\right)\right), \\
v_{\alpha}=\sqrt{\frac{k}{\left|f_{\alpha \alpha}\left(u_{0}, v_{0}\right)\right|}\left|f_{\alpha}\left(u_{0}, v_{0}\right)\right|}
\end{gathered}
$$

and function $F$ is the Fresnel integral, $F_{s}\left(v_{\alpha}\right)=\int_{v_{\alpha}}^{\infty} \mathrm{e}^{\mathrm{j} s t^{2}} \mathrm{~d} t$.

3. Vertex points, these are defined as the four corner points of the curve bounding the domain of integration. Their contribution to the integral is

$$
\boldsymbol{I}=-(-1)^{u_{0}+v_{0}} \mathrm{e}^{\mathrm{j} k f\left(u_{0}, v_{0}\right)} \frac{\boldsymbol{G}\left(u_{0}, v_{0}\right)}{k^{2} f_{u}\left(u_{0}, v_{0}\right) f_{v}\left(u_{0}, v_{0}\right)} .
$$

When vertex points are close to the first kind of critical points or to boundary critical points, it is necessary to consider the coupling effect. Then (31) should be modified as

$$
\begin{aligned}
\boldsymbol{I}= & -4(-1)^{u_{0}+v_{0}} \mathrm{e}^{\mathrm{j} k f\left(u_{0}, v_{0}\right)} \boldsymbol{G}\left(u_{0}, v_{0}\right) \\
& \frac{\operatorname{sign}\left(k f_{u}\left(u_{0}, v_{0}\right)\right) \operatorname{sign}\left(k f_{v}\left(u_{0}, v_{0}\right)\right)}{\sqrt{\left|k f_{u u}\left(u_{0}, v_{0}\right)\right|\left|k f_{v v}\left(u_{0}, v_{0}\right)\right|}} T_{u} T_{v}
\end{aligned}
$$

where $T_{\alpha}=\mathrm{je} \mathrm{e}^{-\mathrm{j} s v_{\alpha}^{2}} \operatorname{sign}\left(k f_{\alpha \alpha}\left(u_{0}, v_{0}\right)\right) F_{s}\left(v_{\alpha}\right)$.

Once each kind of contribution is calculated, to find the total value of the PO integral final summation of all contributions is necessary.

According to PO approach, current is only calculated on the illuminated regions, therefore, for the shadowed regions the current is assigned directly as zero. Since NURBS modeled structures may include large surfaces, there may be some parts of the structure that are partially illuminated. As a result, three different surfaces can be obtained: 1) totally illuminated, 2) totally shadowed, and 3) partially illuminated. For partially illuminated surfaces, the 
shadow boundaries are determined by subdividing the surface in halves for both dimensions $u$ and $v$.

\section{Numerical Results}

In this section comparison between numerical and asymptotic methods are shown for two different structures, where an antenna is positioned near or on top of them. All the simulations have been performed with a $2.8 \mathrm{GHz}$ processor PC. For the simulation and modeling of structures MATLAB R2016a and a computer aided geometric design (CAGD) program is used.

Three different cases are investigated in this section. For the first case three methods are implemented: 1) MoM, 2) conventional hybrid MoM-PO method meshed by triangular facets and 3) presented hybrid MoM-PO method. For the second and third cases, only second and third methods are implemented. Throughout the simulations MoM calculations are used with RWG triangular patch basis functions. For triangular patch modeling size of the triangular facet is $0.13 \lambda$. The width and height of the antennas used in each example is taken as $0.001 \mathrm{~m}$. Besides that, the lengths are expressed for each case in the following paragraphs.

In the first case a curvy plate, which is a section of a sphere with a radius of $0.6 \mathrm{~m}$ is used. The plate covers the angular region $0^{\circ}<\varphi<90^{\circ}$ and $45^{\circ}<\varphi<135^{\circ}$. A dipole antenna with a length of $0.3 \mathrm{~m}$ works at $1 \mathrm{GHz}$ frequency and is located at $(0.7424,0.7424,0)$. It is $0.45 \mathrm{~m}$ away from the surface and perpendicular to $x y$-plane. Since the antenna is at least $1.5 \lambda$ away from the surface, the entire structure constitutes the PO-region.

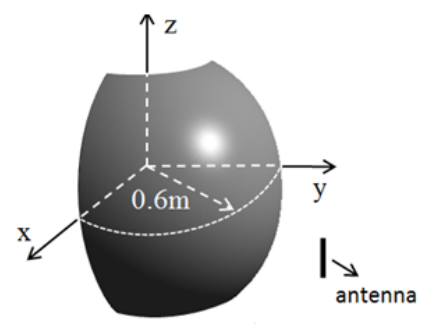

Fig. 4. Antenna around a curvy plate.

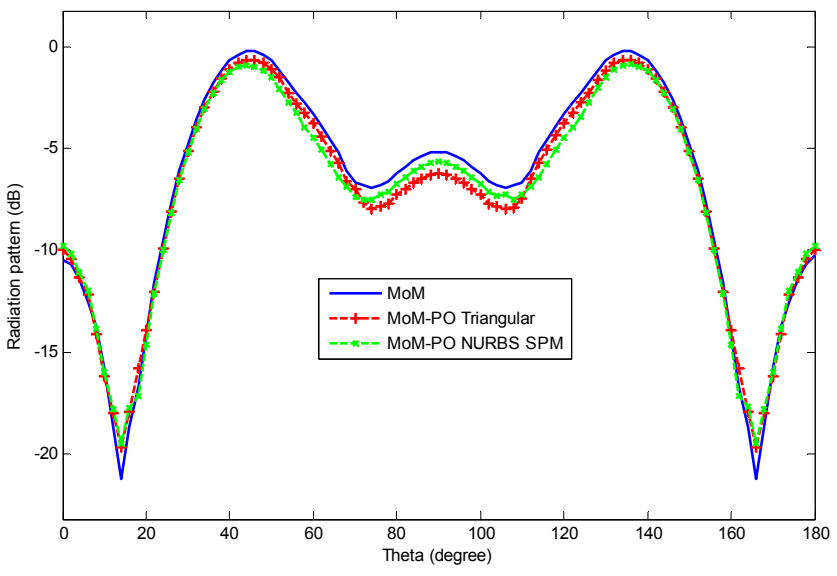

Fig. 5. Radiation pattern of $\varphi<45^{\circ}$ plane.
The radiation pattern of $\varphi<45^{\circ}$ plane is calculated and shown in Fig. 5. Compared to triangular facet based MoM-PO, the results obtained from the presented SPMNURBS based MoM-PO has values closer to MoM between $78^{\circ}$ to $102^{\circ}$. On the other hand, between the degrees of $44^{\circ}$ to 68 and $^{\circ} 112^{\circ}$ to $136^{\circ}$ it gives worse results with a difference of $0.55 \mathrm{~dB}$. Maximum difference between the results of MoM and presented method is $1.7 \mathrm{~dB}$ at $14^{\circ}$ and $166^{\circ}$. The analysis with the MoM and conventional triangular based hybrid method took 41 mins $22 \mathrm{~s}$ and $45 \mathrm{~s}$, respectively. Whereas, the presented hybrid method needs $9 \mathrm{~s}$. The number of unknowns for purely MoM calculation is 1232. For conventional triangular MoM-PO method, number of unknowns in the MoM-region is 14 and in the PO-region it is 1218.

Comparing the presented method with MoM validates the accuracy of the presented hybrid method. Nevertheless, the obtained result can be compared with a published work for further confirmation. In [16] a dipole is positioned in front of a metallic sphere and radiation pattern is obtained by MoM. As the structure used in the first case is a section of a sphere, the $-90^{\circ}<\varphi<90^{\circ}$ part of the radiation pattern obtained in [16] should be taken into account. It can be seen that the results are in close proximity. Comparison of each method in terms of computation time and number of unknowns are given in Tab. 1.

Depending on the size of the structure, when a curvy object is modeled with triangular facets, too many facets need to be used in order to provide the accuracy. It is important to emphases that for triangular modeled simulations, the size of triangles are checked for convergence.

In the second case, a square metallic plate with a side length of $2 \mathrm{~m}$ is considered. As seen in Fig. 6, one of its edges is positioned to the origin and a $0.15 \mathrm{~m}$ dipole antenna is placed perpendicular and on top of its center. The distance between the plate and the antenna is $0.15 \mathrm{~m}(\lambda / 2)$.

\begin{tabular}{|c|c|c|}
\hline Method & $\begin{array}{c}\text { Number of } \\
\text { Unknowns }\end{array}$ & $\begin{array}{c}\text { Total CPU Time } \\
{[\mathrm{sec}]}\end{array}$ \\
\hline MoM & 1232 & 2564 \\
\hline MoM-PO Triangular & $1232(14+1218)$ & 45 \\
\hline MoM-PO NURBS SPM & $14+$ NURBS & 9 \\
\hline
\end{tabular}

Tab. 1. Comparison of the different methods for the first case.

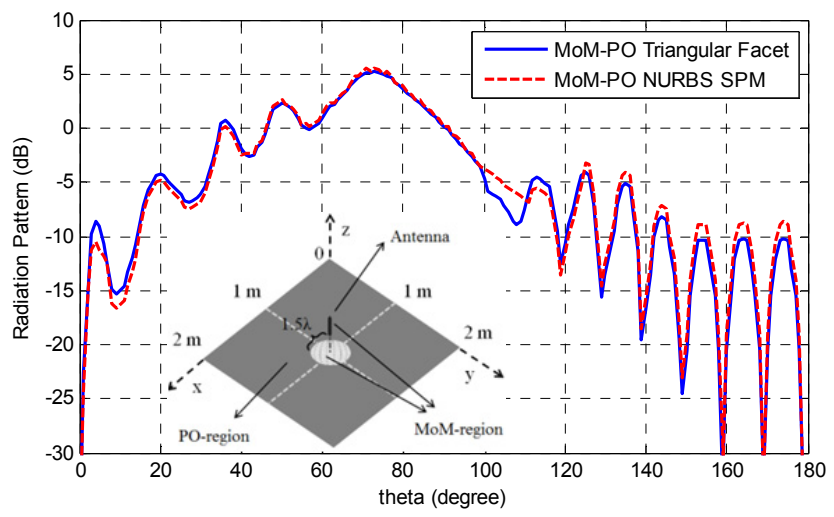

Fig. 6. Vertical radiation pattern of the antenna on top the plate. 
Antenna and parts of the structure, that are as close as $0.45 \mathrm{~m}(1.5 \lambda)$ to the antenna, are taken as the MoM-region. The MoM-region of the structure is a circle with a radius of $0.42 \mathrm{~m}\left(\sqrt{0.45^{2}-0.15^{2}}\right)$. The vertical radiation patterns, obtained by using triangular facet modelled conventional MoM-PO and the proposed hybrid method are calculated for $2 \mathrm{GHz}$ and shown in Fig. 6.

A similar square plate and scheme is used in [16], [21], where the number of unknowns are 1220 and the results are obtained by using MoM. The accuracy of the presented method can be determined by comparing the results in Fig. 6 and the ones observed in [16], [21]. For the results that are in close proximity, better computation times are achieved, as can be seen in Tab. 2 .

In the third case, a monopole antenna is mounted on top of an unmanned aerial vehicle (UAV). The length and diameter of the fuselage is $9 \mathrm{~m}$ and $1.4 \mathrm{~m}$, respectively. The wings are connected from the center of the fuselage with an angle of $60^{\circ}$. The length and width of the wings are $3.6 \mathrm{~m}$ and $0.8 \mathrm{~m}$, respectively. The antenna has a length of $0.3 \mathrm{~m}$ and is located vertically to the middle of the fuselage with respect to $\mathrm{x}$ and $\mathrm{y}$-axis. The frequency is selected as $1 \mathrm{GHz}$.

The antenna and the parts of the structure that are within one and a half wavelengths away to the antenna constitute the MoM-region as seen in Fig. 7. This region is modeled with triangular patches and moment method is used. Sub-domain basis functions are used for the antenna. This matters; because it impacts the way shadowing enters the simulation. For example in Fig. 7 a small current element near the base of the monopole does not illuminate the most of the fuselage, but a current element at the top of the monopole illuminates a large part of the fuselage. The number of unknowns for the MoM-region is 748. The parts of the structure that are more than one and a half wavelengths away from the antenna are considered as PO-region. The PO integral in this region is calculated with SPM, which is a frequency independent method, over Bézier surfaces. As mentioned at the end of Sec. 2, subdivision is applied for the partially illuminated surfaces in the POregion.

Due to the electrical size of the current structure, calculating radiation patterns with traditional MoM takes hours. That is why traditional MoM is not considered; only triangular facet modeled MoM-PO and presented method are compared in this case.

The radiation patterns of the antenna in polar form are given in Fig. 8 and Fig. 9, for the planes $\varphi=90^{\circ}$ and $\varphi=45^{\circ}$, respectively. The waveforms are computed with the angular range from $0^{\circ}$ to $360^{\circ}$ with step size of $2^{\circ}$. For triangular facet based MoM-PO, the entire surface is mod-

\begin{tabular}{|c|c|c|}
\hline Method & $\begin{array}{c}\text { Number of } \\
\text { Unknowns }\end{array}$ & $\begin{array}{c}\text { Total CPU Time } \\
{[\mathrm{sec}]}\end{array}$ \\
\hline MoM used in [16] & 1220 & 188 \\
\hline MoM-PO Triangular & 5002 & 107 \\
\hline MoM-PO NURBS SPM & $624+$ NURBS & 27 \\
\hline
\end{tabular}

Tab. 2. Comparison of the different methods for the $2^{\text {nd }}$ case.

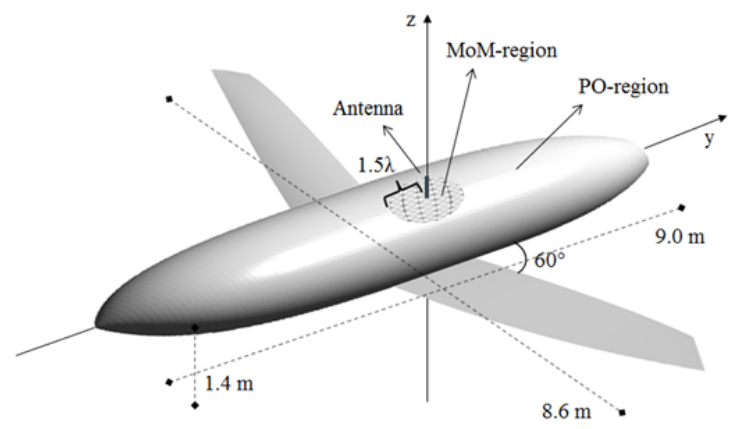

Fig. 7. MoM-PO-region distribution for a UAV with an antenna mounted on top.

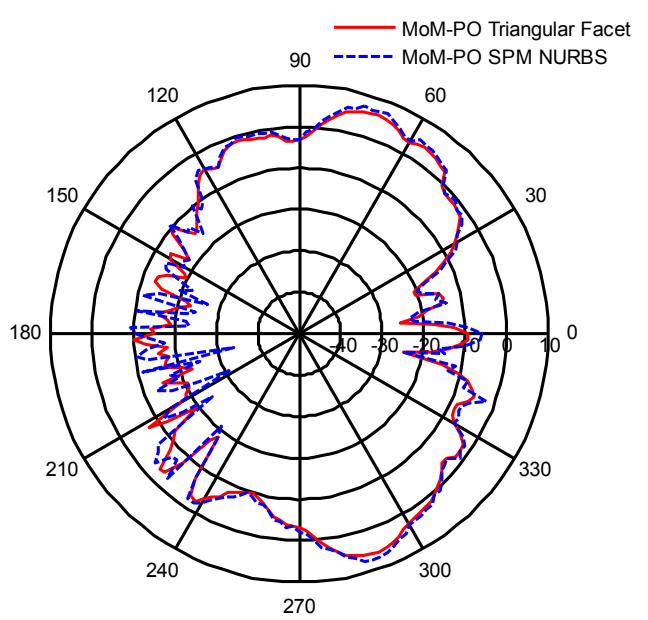

Fig. 8. Radiation pattern of the monopole on the airplane for $\varphi=90^{\circ}$ plane.

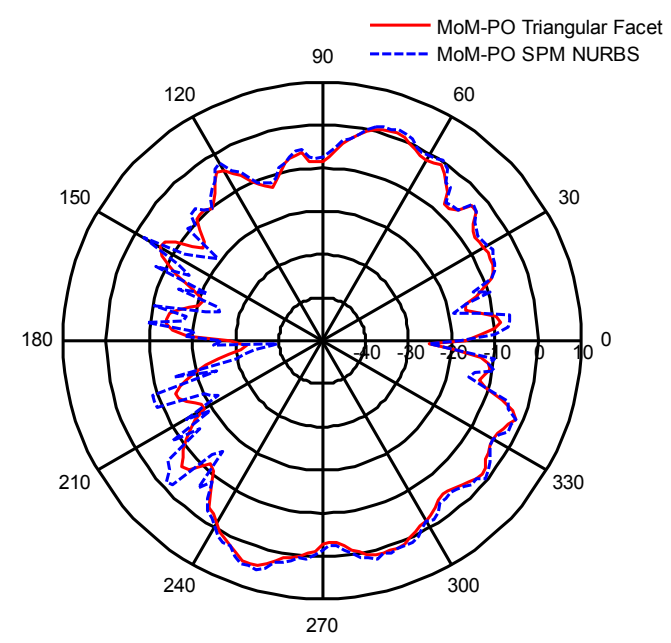

Fig. 9. Radiation pattern of the monopole on the airplane for $\varphi=45^{\circ}$ plane.

eled with 52412 triangular patches. As can be seen from Fig. 8 and Fig. 9, the waveforms derived from the presented method compare well with the ones obtained by triangular facet based MoM-PO. Comparing these curves indicates deviations for back lobes, especially for $\varphi=90^{\circ}$ cut. For the presented SPM-NURBS based MoM-PO method some surfaces are partially illuminated. The deviations are mainly due to this shadowing and the fact that 
diffractions on the edges are not considered in PO approximation.

When the radiation patterns obtained for $\varphi=90^{\circ}$ and $\varphi=45^{\circ}$ planes are analyzed, it can be observed that the antenna gains in the side lobes are similar in each plane. On the other hand for $\varphi=45^{\circ}$ plane, antenna gain in the main lobe is greater; in the back lobe it is smaller in comparison to $\varphi=90^{\circ}$ plane.

The overall computational time required for the solution by triangular facet based MoM-PO and presented method are $7 \mathrm{~min} 38 \mathrm{~s}$ and $1 \mathrm{~min} 20 \mathrm{~s}$, respectively. In other words, the presented MoM-PO method is much more efficient.

To validate the accuracy and the efficiency of the presented method, results can be compared with the ones obtained in [22]. The length, width and height of the airplane used in [22] are $30 \mathrm{~m}, 22 \mathrm{~m}$ and $4 \mathrm{~m}$, respectively. Although that airplane has bigger dimensions, the total number of unknowns taken in that example is 7326 . The comparison between each method can be given in Tab. 3 . A dramatic decrease in computation time is observed between the MoM used in [22] and the presented hybrid method.

\begin{tabular}{|c|c|c|}
\hline Method & $\begin{array}{c}\text { Number of } \\
\text { Unknowns }\end{array}$ & $\begin{array}{c}\text { Total CPU Time } \\
{[\mathrm{sec}]}\end{array}$ \\
\hline MoM used in [22] & 7326 & 16680 \\
\hline MoM-PO Triangular & 52412 & 458 \\
\hline MoM-PO NURBS SPM & $748+$ NURBS & 80 \\
\hline
\end{tabular}

Tab. 3. Comparison of the different methods for the third case.

The simulation results given above are obtained either for $1 \mathrm{GHz}$ or $2 \mathrm{GHz}$ frequency. It is possible to perform the numerical analysis for higher frequency values. But, if the results derived from the hybrid NURBS SPM based MoMPO method are in good agreement with these frequency values then the results should be even better at higher frequencies. Since SPM is a method which is independent on frequency, an increase in the operating frequency does not affect the computation time for PO-region. On the other hand, a frequency increase causes an increase in time for MoM-region. Therefore, the smaller the MoM-region, the less frequency dependent the computation time is.

To analyze the effect of frequency change in computation time, numerical analyses are performed for a variety of higher frequencies. The results derived for the first and third cases are given in Tab. 4 and Tab. 5, respectively. An increase in the frequency causes more change for the third case than the first case; since the number of unknowns in the MoM-region is higher.

The PO current integral is calculated by using SPM. To further reduce the computation time, fast locating algorithms [20] can be used to determine the critical points in SPM. In addition to that, MoM calculations can also be made over NURBS surfaces. If the MoM-region is also modelled by NURBS surfaces instead of triangle facets, that can also decrease the computation time.

\begin{tabular}{|c|c|c|c|}
\hline Method & $\begin{array}{c}\text { Number of Unknowns } \\
\text { (MoM-region+PO-region) }\end{array}$ & Frequency & $\begin{array}{c}\text { Total CPU } \\
\text { Time [sec] }\end{array}$ \\
\hline & & $1 \mathrm{GHz}$ & 9 \\
\cline { 3 - 4 } MoM-PO & \multirow{3}{*}{$\begin{array}{c}\text { NURBS } \\
\text { SPM }\end{array}$} & $14+\mathrm{GHz}$ & 9 \\
\cline { 3 - 4 } & & $4 \mathrm{GHz}$ & 9 \\
\cline { 3 - 4 } & & $10 \mathrm{GHz}$ & 11 \\
\cline { 3 - 4 } & & $20 \mathrm{GHz}$ & 11 \\
\hline
\end{tabular}

Tab. 4. Comparison of the results obtained for the first case

\begin{tabular}{|c|c|c|c|}
\hline Method & $\begin{array}{c}\text { Number of Unknowns } \\
\text { (MoM-region+PO-region) }\end{array}$ & Frequency & $\begin{array}{l}\text { Total CPU } \\
\text { Time [sec] }\end{array}$ \\
\hline \multirow{6}{*}{$\begin{array}{c}\text { MoM-PO } \\
\text { NURBS } \\
\text { SPM }\end{array}$} & \multirow{6}{*}{$748+$ NURBS } & $1 \mathrm{GHz}$ & 80 \\
\hline & & $2 \mathrm{GHz}$ & 80 \\
\hline & & $4 \mathrm{GHz}$ & 84 \\
\hline & & $10 \mathrm{GHz}$ & 92 \\
\hline & & $20 \mathrm{GHz}$ & 97 \\
\hline & & $50 \mathrm{GHz}$ & 103 \\
\hline
\end{tabular}

Tab. 5. Comparison of the results obtained for the third case

For structures that have smooth surfaces, the effect of diffractions can be neglected. But for complex shaped objects, there is a difference over the scattered electric field. If methods like PTD [19], GTD or uniform theory of diffraction (UTD) [10] are used more accurate results can be obtained especially at the edges and corners.

\section{Conclusions}

A hybrid EM computation technique based on the full-wave MoM and the PO approximation is described to analyze antennas mounted on structures. SPM has been applied for the PO integral of induced current on the surface which is modeled by NURBS surfaces. This makes the PO approximation part independent on the operating frequency. One important issue is that, since SPM is invalid when the distance between the observation point and the structure is less than one and a half wavelength, the observation point is always considered to be far from the structure.

The computational efficiency of the presented method depends on the type of a problem as well as on how the structure is subdivided into MoM and PO-regions. Because if the object is not electrically large or if the MoM-region is much larger than PO-region, then the efficiency for both CPU time and memory storage decreases.

Numerical examples illustrate the accuracy and most importantly the efficiency of this method, compared to single MoM and MoM-PO method based on totally triangular patching.

\section{References}

[1] LACIK, J., RAIDA, Z. Modeling microwave structures in time domain using Laguerre polynomials. Radioengineering, 2006, vol. 15 , no. 3 , p. 1-9. ISSN: $1210-2512$ 
[2] RAO, S., WILTON, D., GLISSON, A. Electromagnetic scattering by surfaces of arbitrary shape. IEEE Transactions on Antennas and Propagation, 1982, vol. 30, no. 3, p. 409-418. DOI: 10.1109/TAP.1982.1142818

[3] SONG, J., LU, C., CHEW, W. C. Multilevel fast multipole algorithm for electromagnetic scattering by large complex objects. IEEE Transactions on Antennas and Propagation, 1997, vol. 45, no. 10 , p. $1488-1493$. DOI: $10.1109 / 8.633855$

[4] Schejbal, V., SVOBOda, P., PIDANic, J., et al. Twodimensional forward scattering comparisons of approximate and exact solutions. Radioengineering, 2012, vol. 21, no. 1, p. 471 to 477. ISSN: $1210-2512$

[5] CERNY, O., DOLECEK, R., KOPECKY, P., et al. Optimization of far-field antenna range. Radioengineering, 2015, vol. 24, no. 4, p. 892-897. DOI: 10.13164/re.2015.0892

[6] GORDON, W. B. Far-field approximation to the KirchoffHelmholtz representations of scattered field. IEEE Transactions on Antennas and Propagation, 1975, vol. 23, p. 590-592. DOI: 10.1109/TAP.1975.1141105

[7] LUDWIG, A. Computation of radiation patterns involving numerical double integration. IEEE Transactions on Antennas and Propagation, 1968, vol. 16, no. 6, p. 767-769. DOI: 10.1109/TAP.1968.1139296

[8] FILON, L. N. G. On a quadrature formula for trigonometric integrals. Proceedings of the Royal Society of Edinburgh, 1928, vol. 49, p. 38-47. DOI: $10.1017 /$ S0370164600026262

[9] LEVIN, D. Procedures for computing one and two dimensional integrals of functions with rapid irregular oscillations. Mathematics of Computation, 1982, vol. 38, no. 158, p. 531-538. DOI: 10.1090/S0025-5718-1982-0645668-7

[10] BONDIA-VICO, F., FERRANDO-BATALLER, M., VALERONOGUEIRA, A. A new fast physical optics for smooth surfaces by means of a numerical theory of diffraction. IEEE Transactions on Antennas and Propagation, 2010, vol. 58, no. 3, p. 773-789. DOI: 10.1109/TAP.2009.2039308

[11] WU, Y. M., JIANG, L. J., SHA, W. E. I., CHEW, W. C. The numerical steepest descent path method for calculating physical optics integrals on smooth conducting quadratic surfaces. IEEE Transactions on Antennas and Propagation, 2013, vol. 61, no. 8, p. 4183-4193. DOI: 10.1109/TAP.2013.2259788

[12] PEREZ, J., CATEDRA, M. F. Application of physical optics to the RCS computation of bodies modeled with NURBS surfaces. IEEE Transactions on Antennas and Propagation, 1994, vol. 42, no. 10, p. 1404-1411. DOI: $10.1109 / 8.320747$

[13] CONDE, O. M., PEREZ, J., CATEDRA, M. F. Stationary phase method application for the analysis of radiation of complex 3-D conducting structures. IEEE Transactions on Antennas and Propagation, 2001, vol. 49, no. 5, p. 724-731. DOI: $10.1109 / 8.929626$

[14] ZHANG, J., YU, W. M., ZHOU, X. Y., et al. Efficient evaluation of the physical-optics integrals for conducting surfaces using the uniform stationary phase method. IEEE Transactions on Antennas and Propagation, 2012, vol. 60, no. 5, p. 2398-2408. DOI: 10.1109/TAP.2012.2189737

[15] GUAN, Y., GONG, S., XU, Y., et al. Computation of RCS of targets modelled with trimmed NURBS surfaces. IET Electronics Letters, 2009, vol. 45, no. 21, p. 1092-1093. DOI: $10.1049 / \mathrm{el} .2009 .1449$

[16] JAKOBUS, U., LANDSTORFER, F. M. Improved PO-MM hybrid formulation for scattering from three-dimensional perfectly conducting bodies of arbitrary shape. IEEE Transactions on Antennas and Propagation, 1995, vol. 43, no. 2, p. 162-169. DOI: $10.1109 / 8.366378$

[17] HODGES, R. E., RAHMAT-SAMII, Y. An iterative current-based hybrid method for complex structures. IEEE Transactions on Antennas and Propagation, 1997, vol. 45, no. 2, p. 265-276. DOI: $10.1109 / 8.560345$

[18] VAlle, L., RIVAS, F., CATEDRA, M. F. Combining the moment method with geometrical modeling by NURBS surfaces and Bezier patches. IEEE Transactions on Antennas and Propagation, 1994, vol. 42, no. 3, p. 373-381. DOI: $10.1109 / 8.280724$

[19] ADANA, F. S., DIEGO, I. G., BLANCO, O. G., et al. Method based on physical optics for the computation of the radar cross section including diffraction and double effects of metallic and absorbing bodies modeled with parametric surfaces. IEEE Transactions on Antennas and Propagation, 2004, vol. 52, no. 12, p. 3295-3303. DOI: 10.1109/TAP.2004.836444

[20] LIU, J., GUO, L. Evaluation of physical optics integrals from bspline surfaces by means of a fast locating algorithm of stationary points. IEEE Transactions on Antennas and Propagation, 2017, vol. 65, no. 3, p. 1495-1499. DOI: 10.1109/TAP.2016.2640146

[21] DJORDJEVIC, M., NOTAROS, B. M. Higher order hybrid method of moments-physical optics modelling technique for radiation and scattering from large perfectly conducting surfaces. IEEE Transactions on Antennas and Propagation, 2005, vol. 53, no. 2, p. 800-813. DOI: 10.1109/TAP.2004.841318

[22] NOGA, A., KARWOWSKI, A. Analysis of electrically large problems using the hybrid MM-PO method. In MIKON 2008 17th International Conference on Microwaves, Radar and Wireless Communications. Wroclaw (Poland), May 2008. ISBN: 978-83-906662-8-0

\section{About the Authors ...}

Tayfun OKAN (corresponding author) was born in Ankara in 1988. He received his B.S. degree in Electrical and Electronics Engineering from Başkent University and the M.S. degree in Electrical-Electronic Engineering from Gazi University, Ankara, in 2012 where he is currently pursuing his $\mathrm{Ph} . \mathrm{D}$. degree in the same department. He is working as a lecturer at the University of Turkish Aeronautical Association. His research interests concentrate on radar signal processing and computational electromagnetics.

Nursel AKÇAM was born in Ardahan, Turkey, 1965. She received the M.S. and Ph.D. degrees in Electrical and Electronics Engineering from the University of Gazi, Ankara, Turkey, in 1993 and 2001, respectively. From 1987 to 2002, she was a Research Assistant with the Electromagnetic Theory, and Microwave Technique Laboratory, University of Gazi. Since 2016, she has been an associate professor at the same University. She is the author of over 50 articles. Her research interests include asymptotic high frequency methods, numerical methods in electromagnetic theory, blocking aperture in reflector antennas, communication theory, and spread spectrum and radar systems. 